\title{
Sliding Mode Control for 2 Degrees of Freedom Upper Limb Rehabilitation Robotic System under Uncertainties
}

\author{
Syed Faiz Ahmed, Yarooq Raza, Abadal Salam T. Hussian, M. Kamran Joyo, Asadullah Shah
}

\begin{abstract}
Rehabilitation of patients suffering from post-stroke injuries via robots is now adapted word widely. The aim of this therapy is to restore and improve the dysfunction and the performance of the affected limbs doing repetitive tasks with the help of rehabilitation robots, as robots are best way to perform repetitive task without any monotony failure. Control of these rehabilitation robots is an important part to consider because of nonlinearity and uncertainty of the system. This paper presents nonlinear sliding mode controller (SMC) for controlling a 2 degrees of freedom (DOF) upper limb robotic manipulator. Sliding mode control is able to handle system uncertainties and parametric changes. One drawback of using SMC is high frequency oscillations called as chattering. This chattering can be reduced by using boundary layer technique. Experiments have been carried out under perturbed conditions and results have shown that SMC performs well and remain stable and thus proves to robust controller for upper limb robotic manipulator.
\end{abstract}

Keywords : Rehabilitation robots; Non-linearities; Sliding Mode Control; Chattering; Boundary Layer Technique; Perturbed Condition.

\section{INTRODUCTION}

Stroke, the sudden death of certain brain cells [1] is the third leading reason behind disability and the second fatal cause among the people globally [2] especially in elderly people. Life expectancy is continuing to improve which means that the number of aged persons will be growing in the next few decades and consequences will be increasing number of stroke patients. Rehabilitation of these patients continues generally throughout their lifetime. For the reason, this long-lasting therapy requires men power which turns this into a costly program. For a person, ability to move is vital in its daily life activities. Especially most of the work is done by using our hands, hence disorders of upper extremities limit

Revised Manuscript Received on December 30, 2019.

* Correspondence Author

Syed Faiz Ahmed*, Kulliyyah of Information \& Communication Technology International Islamic University Malaysia, Kuala Lumpur, Malaysia, syedfaiz@pmail.ntu.edu.sg

Yarooq Raza, Department of Electrical/ Electronics, University Kuala Lumpur, Kuala Lumpur, Malaysia, syed.yarooq@s.unikl.edu.my.

Abadal salam T. Hussian, Medical Instrumentations Techniques Engineering, AlKitab University, Iraq

M. Kamran Joyo, Department of Electrical/ Electronics, University Kuala Lumpur, Kuala Lumpur, Malaysia, muhammad.kamran@s.unikl.edu.my

Asadullah Shah, Kulliyyah of Information \& Communication Technology International Islamic University Malaysia, Kuala Lumpur, Malaysia, asadullah@iium.edu.my

(C) The Authors. Published by Blue Eyes Intelligence Engineering and Sciences Publication (BEIESP). This is an open access article under the CC BY-NC-ND license (http://creativecommons.org/licenses/by-nc-nd/4.0/) the independence of life and yet reducing life quality. There exist many approaches that can help in restoring the functionalities of the upper limb extremities such as physical therapy [3], functional electrical stimulation and orthoses. Fruitful outcome of such physical therapy based on duration type and intensity of training, attention of physiotherapists and health condition of patients [4-6]. After finishing acute therapy for stroke, a continued improvement in patients have been found suggesting that rehabilitation shall continue for several years post stroke attack. With the increasing number of stroke patients, rehabilitation have become more and more difficult because of labor-intensive nature of treatment. Rehabilitation robots have the potential to meet this increasing demand that conventional therapy is facing [7]. Since these treatments require repetitive tasks, robots are the best way that can be used as they do not face fatigue failure and monotony [8]. These robots are capable to treat the patient in the absence of physiotherapists and offer more frequent treatment as well as dropping the costs [9].

These rehabilitation robots need a stable and robust controller for providing safer, reliable and comfortable exercises to the suffering ones, which involves moving patient's hand through a pre-determine trajectory. Many linear and non-linear approaches have been used to control these robots. Linear methods include PID controller [10,11]. But the control strategy for these robots is a bit complex and difficult because of the dynamic model their nonlinear characteristics and the limitations for exact dynamic parameters estimation [12]. Therefore, non-linear controllers are required to deal with such issues, these non-linear controllers include sliding mode controller [13], computed torque control [10] and impedance control [14]. Key requirement for assisting arm movements during rehabilitation is to have high dynamic performance for tracking to maneuver the exoskeleton in a smooth, continuous and effective manner. Linear control methods are limited to cope up with the problems related with nonlinear model of robots. On the other hand, while using computed torque control, dynamic model was simplified by neglecting mass/inertia and centrifugal terms $[10,15]$ which in turns reduces the tracking ability of the controller. Also, these controllers are not able to deal with the uncertainties of the system. Many other works have been proposed including fictious gain [16], fuzzy adaption technique [17] and neural control [18]. However, neural network and fuzzy logic controls suffer from a slow response time as these control techniques require heavy computation [12].

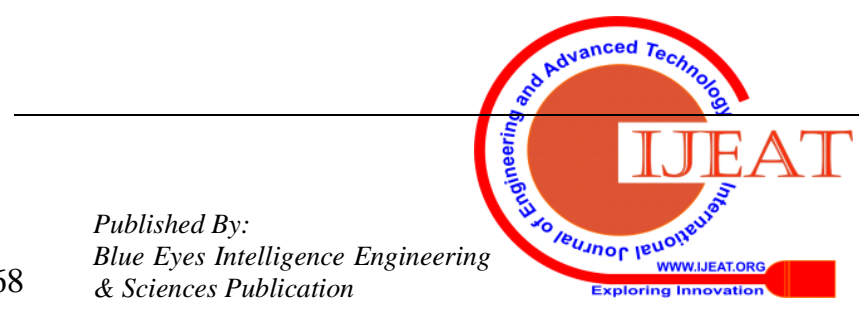




\section{Sliding Mode Control for 2 Degrees of Freedom Upper Limb Rehabilitation Robotic System under Uncertainties}

Sliding mode controller can deals with these issues as it has simple structure, fast transient response and can deals with the uncertainties of the system [19]. SMC deals with bounded disturbances and produces very much little percent overshoot than other controllers such as PID and MPC [34], in the response of the system under disturbed condition and hence maintains stable performance of the system. SMC have been used in many applications such as control of mobile robots [20], actuator control [21] and robotic manipulators [22-25]. One disadvantage of using SMC in practical implementation is chattering. Chattering, arises due to signum function, is high frequency control signal with finite amplitude that produces vibration in the mechanical structure that can cause wear and tear to the components. Hence applying classical SMC to the rehabilitation robots is utterly not suitable for the therapy application as it will cause harm to the patients. Several strategies have been proposed to minimize or eliminate this chattering such as replacing the sign function with a boundary layer function [26] or saturation function [27] and using a continuous hyperbolic tangent function [28]. In this study SMC with boundary layer technique is used for chattering reduction. SMC being model base controller takes into account the dynamic model of the plant, robotic manipulator in our case, for producing output and hence its transient and steady state responses are stable with significant rise time of the system, minimum percent overshoot and with minimum steady state error. For validation of the controller it has been tested under disturbed condition for various strength of disturbance. The disturbances in this work will represent human-arm coupling effects and torque load in practical implementation or physical therapy exercises.

This paper is organized as follows: section II contains system design; Dynamic model is presented in section III, section IV is based on controller design; Experiments and results have been included in section V; section VI contains analysis and discussion; conclusion of the study has been provided in section VII and references are provided at the end of the paper

\section{SYSTEM DESIGN}

The CAD model of the 2 degrees of freedom upper (DOF) arm robot is shown below in Fig. 1 and Fig. 2 presents the kinematics of the robot showing the serial chain of rigid bodies to which attached are frame of references and links lengths are labelled with respective joints. Ultimately the final goal is to predict the position of end effector means frame 2 with respect to base frame 0 . Table I shows the Denavit-Hartenberg (DH) parameters [29] of the 2 DOF upper arm rehabilitation robot.

Table I. Dh Parameters

\begin{tabular}{|l|l|l|l|}
\hline $\boldsymbol{a}_{\mathbf{i}}$ & $\boldsymbol{\alpha}_{\mathbf{i}}$ & $\boldsymbol{d}_{i}$ & $\boldsymbol{\theta}_{\boldsymbol{i}}$ \\
\hline $\boldsymbol{a}_{\mathbf{1}}$ & 0 & 0 & $\boldsymbol{q}_{\mathbf{1}}$ \\
\hline $\boldsymbol{a}_{\mathbf{2}}$ & 0 & 0 & $\boldsymbol{q}_{\mathbf{2}}$ \\
\hline
\end{tabular}

Where; $\alpha_{i}$ is link length, $\alpha_{i}$ and $d_{i}$ is angle twist and offset between frame of references respectively and $\theta_{i}$ is the angle, which is variable.

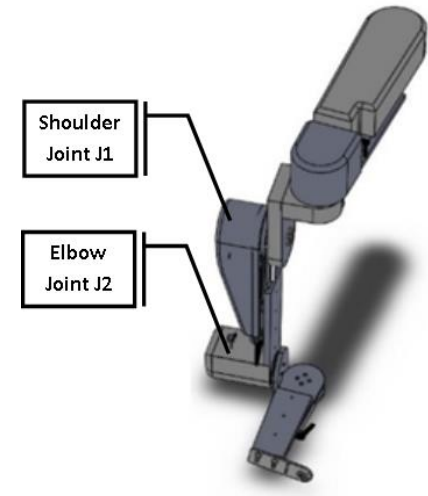

Fig. 1. CAD Model for Upper Limb Robot

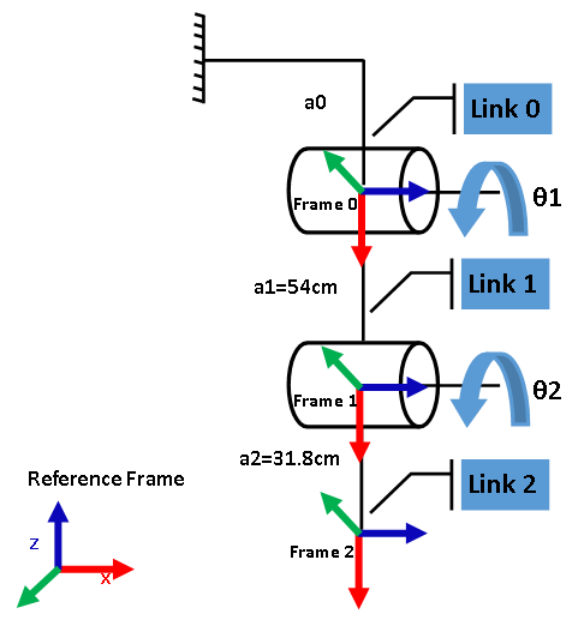

Fig. 2. Kinematics of 2 DOF Upper Limb Robot

$$
\frac{\theta(s)}{E_{a}(s)}=\frac{K}{s(T s+1)}
$$

Here $K_{\tau}$ is motor torque constant, $R_{a}$ is armature resistance, $B$ is viscous friction, $K_{b}$ is motor backlash constant $l$ and is motor inertia.

\section{DYNAMIC MODEL}

Using lagrange-Euler methodology, the robotic dynamics [31] of the of the upper limb robot can be described as following nonlinear differential equation as shown in (2).

$$
M(\theta) \theta_{Y}+F(\theta, \theta) \theta_{Y}+G(\theta)=\tau
$$

Where, $\theta_{v} \theta$ and $\theta$ represents joint position, velocity and acceleration respectively; $\tau$ is driving torques; $M(\theta)$ is inertia matrix; $F(\theta, \theta)$ represents centrifugal matrix; $G(\theta)$ is the gravitational torques.

The model of 2 DOF upper limb robotic manipulator is shown below in (3):

$$
\begin{aligned}
& {\left[\begin{array}{ll}
M_{11} & M_{12} \\
M_{21} & M_{22}
\end{array}\right]\left[\begin{array}{l}
\theta_{1} \\
\theta_{2}
\end{array}\right]+\left[\begin{array}{cc}
-F_{12} \theta_{2} & -F_{12}\left(\theta_{1}+\theta_{2)}\right. \\
F_{12} \theta_{1} & 0
\end{array}\right]\left[\begin{array}{l}
\theta_{1} \\
\theta_{2}
\end{array}\right]+} \\
& {\left[\begin{array}{l}
G_{1} g \\
G_{2} g
\end{array}\right]=\left[\begin{array}{l}
U_{1} \\
U_{2}
\end{array}\right]}
\end{aligned}
$$

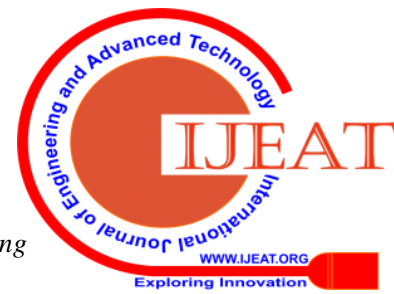


Where;

$$
\begin{aligned}
& M_{11}=\left(m_{1}+m_{2}\right) r_{1}^{2}+m_{2} r_{2}^{2}+2 m_{2} r_{1} r_{2} \cos \left(\theta_{2}\right) \\
& M_{12}=M_{21}=m_{2} r_{2}^{2}+m_{2} r_{1} r_{2} \cos \left(\theta_{2}\right) \\
& M_{22}=m_{2} r_{2}^{2} \\
& F_{12}=m_{2} r_{1} r_{2} \sin \left(\theta_{2}\right) \\
& G_{1}=\left(m_{1}+m_{2}\right) r_{1} \cos \left(\theta_{2}\right)+m_{2} r_{2} \cos \left(\theta_{1}+\theta_{2}\right) \\
& G_{2}=m_{2} r_{2} \cos \left(\theta_{1}+\theta_{2}\right)
\end{aligned}
$$

The initial Conditions are defined as;

$$
\theta_{1}(0)=-80 \text { degree, } \theta_{2}(0)=-70 \text { degree }
$$

Table II shows the parameter values of our upper arm robot model and motor used in the system. Masses and lengths are of mechanical structure and motor parameters is taken form the DC motor model EC-90.

TABLE II. Dynamic Model parameters

\begin{tabular}{|l|l|l|}
\hline Parameters & Shoulder (AB/AD) & Units \\
\hline $\mathbf{K}_{\mathbf{t}}$ & .023 & $\mathrm{~N}-\mathrm{m} / \mathrm{A}$ \\
\hline $\mathbf{R}_{\mathbf{a}}$ & 1 & $\mathrm{Ohm}$ \\
\hline $\mathbf{K}_{\mathbf{b}}$ & 0.023 & $\mathrm{~V}-\mathrm{s} / \mathrm{rad}$ \\
\hline $\mathbf{J}_{\mathbf{m}}$ & 0.02 & $\mathrm{~kg} \cdot \mathrm{m}^{2}$ \\
\hline $\mathbf{b}$ & 0.09 & $\mathrm{~N} \cdot \mathrm{sec} / \mathrm{m}$ \\
\hline Mass upper-arm $\mathbf{( m}_{\mathbf{1}} \mathbf{)}$ & 1.04 & $\mathrm{Kg}$ \\
\hline Mass lower-arm $\left(\mathbf{m}_{\mathbf{2}}\right)$ & 1.86 & $\mathrm{Kg}$ \\
\hline Length upper-arm $\left(\mathbf{r}_{\mathbf{1}}\right)$ & 0.3 & $\mathrm{~m}$ \\
\hline Length lower-arm $\left(\mathbf{r}_{\mathbf{2}}\right)$ & 0.38 & $\mathrm{~m}$ \\
\hline
\end{tabular}

\section{A. Composite Transfer Function}

The composite transfer function of the whole system containing transfer function of motor and joints is stated below in state space form;

For joint 1 and 2 the state space transfer function can be calculated by using following (4),

$$
\begin{aligned}
& \dot{x}(k)=A_{i} x(k)+B_{i} u(k) \\
& y(k)=C_{i} x(k)+D_{i} u(k) \quad(i=1,2)
\end{aligned}
$$

Where $x(k)$ is a state vector, $u(k)$ is an input vector and $y(k)$ as output vector with $A_{\mathrm{i}}, B_{\mathrm{i}}, C_{\mathrm{i}}$ and $D_{\mathrm{i}}$ are the constant state-space matrices of joints 1 and 2, given as follows;

For joint 1;

$$
\begin{gathered}
A_{1}=\left[\begin{array}{cccc}
-2014 & -1029000 & -14400000 & 0 \\
1 & 0 & 0 & 0 \\
0 & 1 & 0 & 0 \\
0 & 0 & 1 & 0
\end{array}\right] \\
B_{1}=\left[\begin{array}{l}
1 \\
0 \\
0 \\
0
\end{array}\right] \\
C_{1}=\left[\begin{array}{llll}
0 & 0 & 0 & 74770000
\end{array}\right] \\
D_{1}=[0]
\end{gathered}
$$

And for Joint 2,

$$
\begin{gathered}
A_{2}=\left[\begin{array}{cccc}
-2050 & -1100000 & -50150000 & 0 \\
1 & 0 & 0 & 0 \\
0 & 1 & 0 & 0 \\
0 & 0 & 1 & 0
\end{array}\right] \\
B_{2}=\left[\begin{array}{l}
1 \\
0 \\
0 \\
0
\end{array}\right] \\
C_{2}=\left[\begin{array}{llll}
0 & 0 & 0 & 89560000
\end{array}\right] \\
D_{2}=[0]
\end{gathered}
$$

\section{CONTROL DESIGN}

\section{A. Sliding Mode Control}

Sliding Mode Control [32] is developed to control 2 DOF upper limb robotic manipulator. The sliding surface of the controller is given below in (5).

$$
s=\lambda e+\dot{e}
$$

Where, $\boldsymbol{\lambda}$ is positive constant and is the slope of sliding surface; $e$ is the error of the closed loop system and $\dot{e}$ is the change in error. The main task is to keep $S$ at zero when the tracking is outside this sliding surface. The control input, $u$ should exist so that:

$$
\frac{1}{2} \frac{d}{d t} S S^{T}<-\eta|S|
$$

Where $\eta$ is a strictly positive constant.

Equation (6) is known as sliding condition [33] [34] and it states that all the Euclidian distances from the sliding surface through all state trajectories is decreasing. This will force the trajectories towards the sliding surface.

The simple solution to get the sliding condition when uncertainty appears is switching control law shown in (7):

$$
\begin{gathered}
u=K \cdot \operatorname{sgn}(s) \\
\operatorname{sgn}(s)= \begin{cases}1 & s>0 \\
-1 & s<0 \\
0 & s=0\end{cases}
\end{gathered}
$$

Here gain $K$ is positive constant. It can be seen from (8) that the control input, $u$ is discontinuous and is not a function of time. This imperfect switching causes chattering. To reduce this chattering [35], a boundary layer is inserted into the controller by replacing the discontinuous signum function $\operatorname{sgn}(s)$ with the linear smooth saturation function $\operatorname{sat}(s / \phi)$ in the control input as shown in (9) and (10):

$$
\begin{gathered}
u=K \cdot \operatorname{sat}(s / \phi) \quad(9) \\
\operatorname{sat}(s / \phi)=\left\{\begin{array}{cc}
1 \text { for } \quad s \geq \phi \\
s / \phi \text { for }-\phi \leq s \leq \phi \quad \forall t, 0<\phi \ll 1 \\
-1 \text { for } \quad s \leq \phi
\end{array}\right.
\end{gathered}
$$

Here $\phi$ is the thickness of the boundary layer.

Table III below shows the values of controller gain, slope and boundary layer thickness of SMC for Joint 1 and 2. These values have been found by hit and trial method.

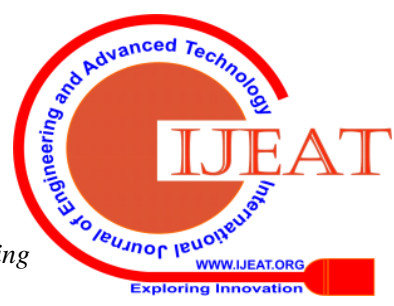


Sliding Mode Control for 2 Degrees of Freedom Upper Limb Rehabilitation Robotic System under Uncertainties

TABLE III. SMC Parameters

\begin{tabular}{|c|c|c|c|}
\hline Joint & $\begin{array}{c}\text { Controller Gain } \\
(\mathbf{K})\end{array}$ & $\begin{array}{c}\text { Slope } \\
(\boldsymbol{\lambda})\end{array}$ & $\begin{array}{c}\text { Boundary Layer Thickness } \\
(\boldsymbol{\phi})\end{array}$ \\
\hline 1 & 10 & 5 & 0.01 \\
\hline 2 & 5 & 5 & 0.01 \\
\hline
\end{tabular}

\section{B. Model Predictive Control}

Model predictive control is an optimization control model-based algorithm. In MPC, current control action is attained by considering past and future behaviour of the plant model. Future values of the control action are optimized online within constraints limits, ensuring best tracking performance. The cost function and constraints can be computed by the optimization process. Two main important parameters of MPC are prediction horizon and control horizon. Numbers of samples predicted is prediction horizon and future trajectory is associated with control horizon.

\section{EXPERIMENTS}

Mathematical model of the 2 DOF upper limb rehabilitation robot is developed using its kinematic model and dynamic model. System transfer function is developed combining motor model and upper arm model. Control law implementation and simulations are carried out by using model. Two controller setups of SMC are established for the two joints. Position output is feedback into the controller as error and derivative of error is being calculated in the SMC box. Disturbances of $50 \%$ and $100 \%$ is applied to the system to verify the performance of the controller under uncertainties. $100 \%$ disturbance means the same amount of disturbance is being applied as the amount of input that is driving the motor and 50\% disturbance is representing half of the input signal given to the motors. The closed loop diagram of the whole system, consisting of input, controller, plant and output, is shown in Fig. 3 and plant simulation setup in MATLAB Simulink for the controller is shown below in Fig. 4. Reference input and robot shown in Fig. 3 are correspondence to step input and joints 1 and 2 in Fig. 4. The disturbance is added in the output of the controller block and the signal is fed to the joints as manipulated variable and error, which is the difference between reference input and output is used as feedback signal to the input a shown in Fig. 4.

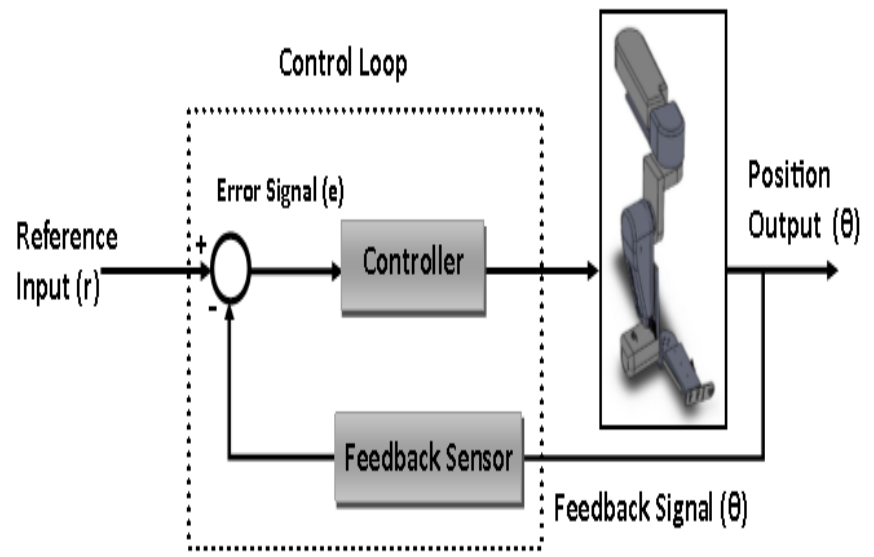

Fig. 3. Closed Loop System Diagram

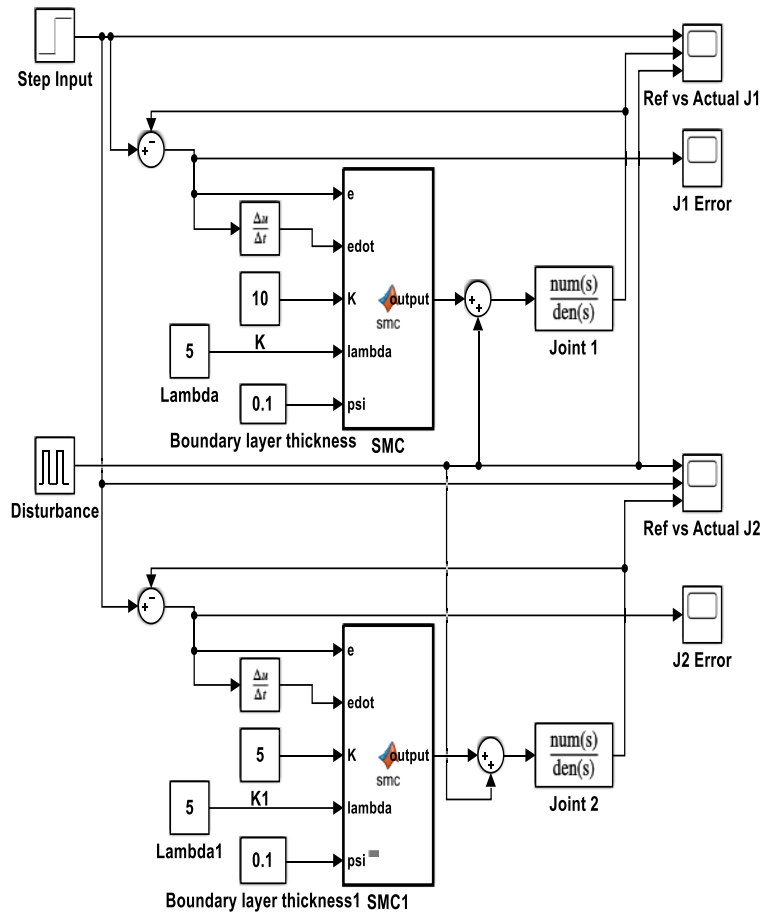

Fig. 4. MATLAB Simulink Model for SMC

\section{A. SMC with Boundary Layer}

To reduce the chattering of SMC, boundary layer of thickness 0.01 is applied to the control input to make the response of the system smooth. Fig. 5 and Fig. 6 show the response graphs for joint 1 and 2 respectively. Fig. 7 and Fig. 8 represents the error graph for joint 1 and 2. Maximum error of 0.0028 at 7.4 second is found in joint 1 and error of 0.0027 at 5.9 seconds is found in joint 2 as shown below.

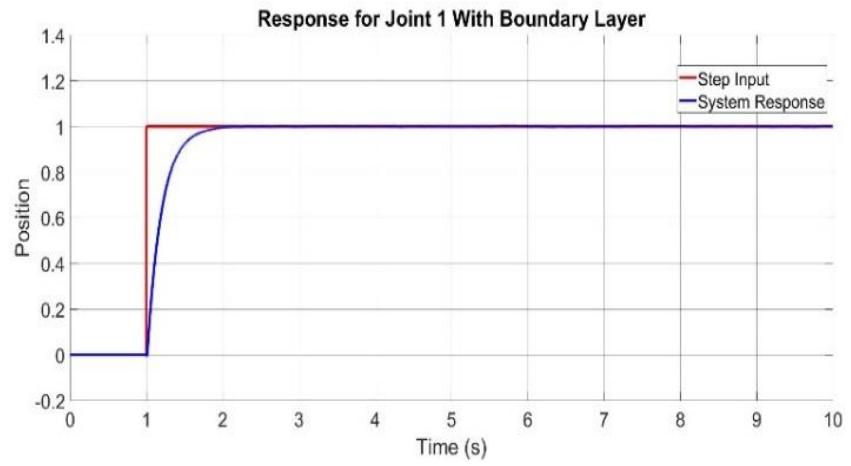

Fig. 5. Joint 1 With Boundary Layer

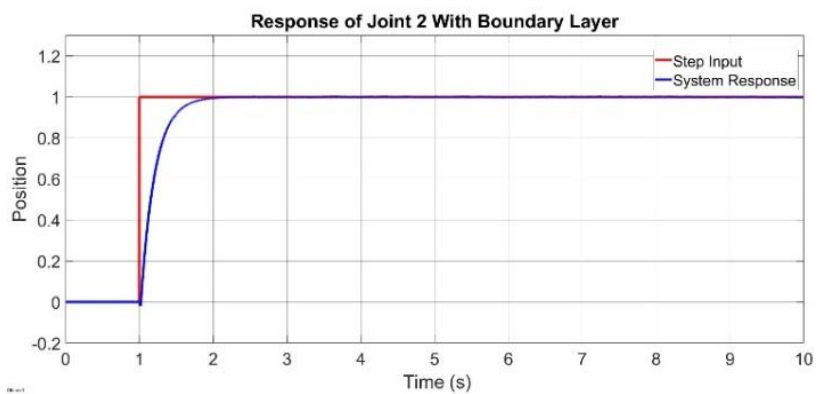

Fig. 6. Joint 2 With Boundary Layer 


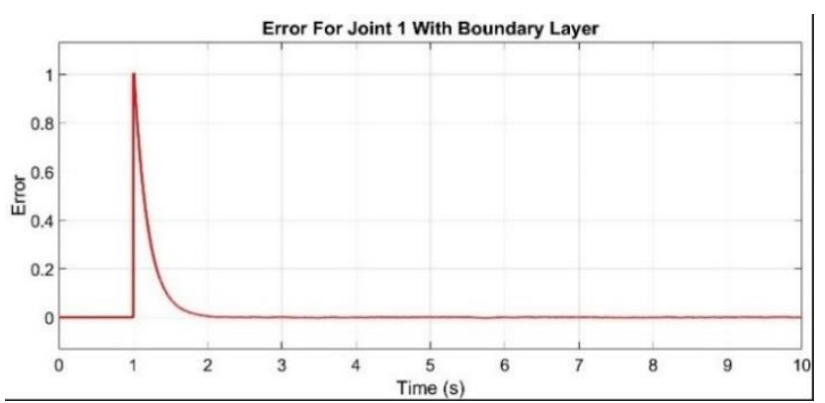

Fig. 7. Error for Joint 1 With Boundary Layer

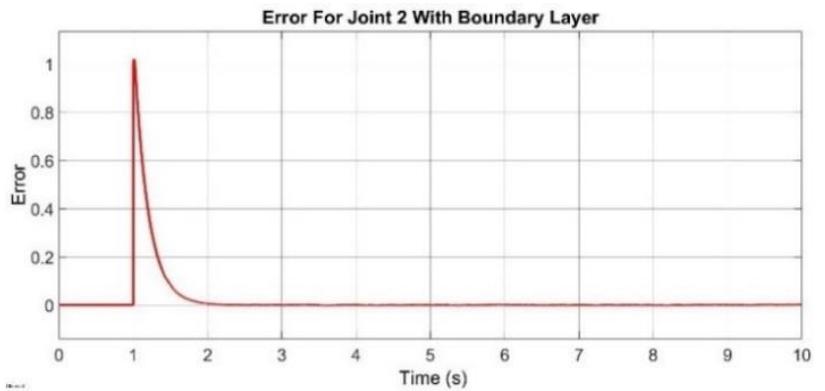

Fig. 8. Error for Joint 2 With Boundary Layer

\section{B. SMC Under $50 \%$ Disturbance}

To check and validate the response of sliding mode control for upper arm robot under perturbed condition, disturbance of $50 \%$ is applied to joint 1 and joint 2and graphs have been plotted. Sliding mode control is found to able to handle the disturbance. Maximum overshoot of joint 1 is found to be 1.007 at 5.3 second and SMC settles the response within 0.83 seconds as shown in Fig. 9. Maximum steady state error of 0.00088 occurs at 6.4 second as shown in Fig. 10. For joint 2 maximum overshoot is approximately equal to 1.09 at 5.28 seconds. The maximum steady state error of 0.00081 found at 7.7 seconds with the settling time of 1.09 seconds as shown in Fig. 11 and 12.

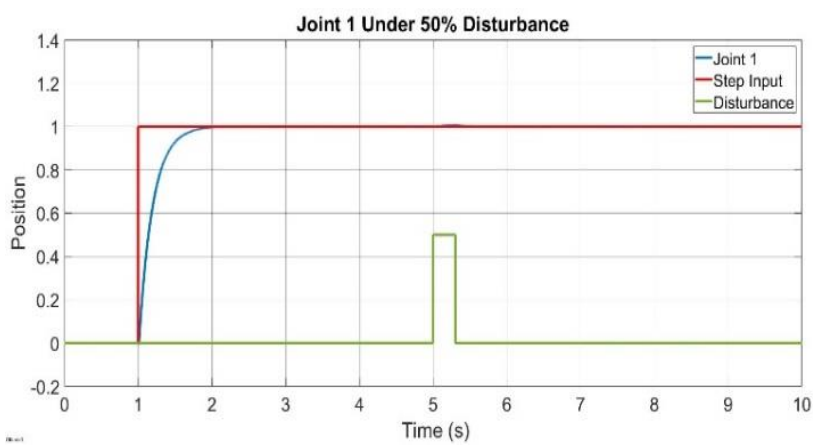

Fig. 9. SMC Under 50\% Disturbance

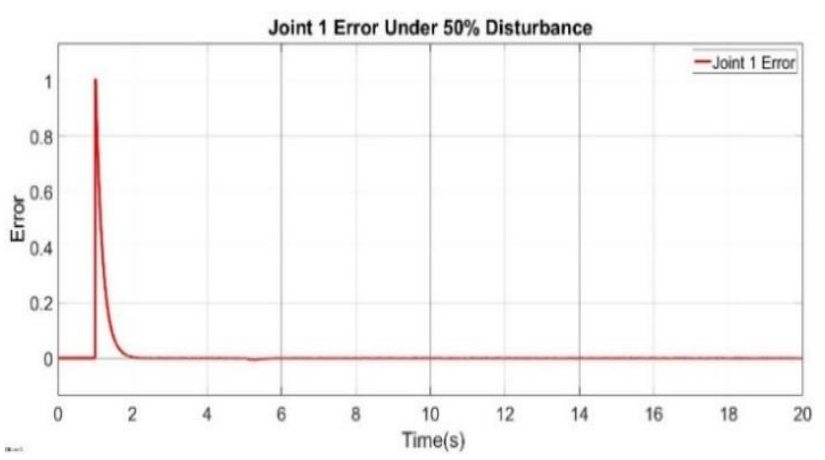

Fig. 10. Error for Joint 1

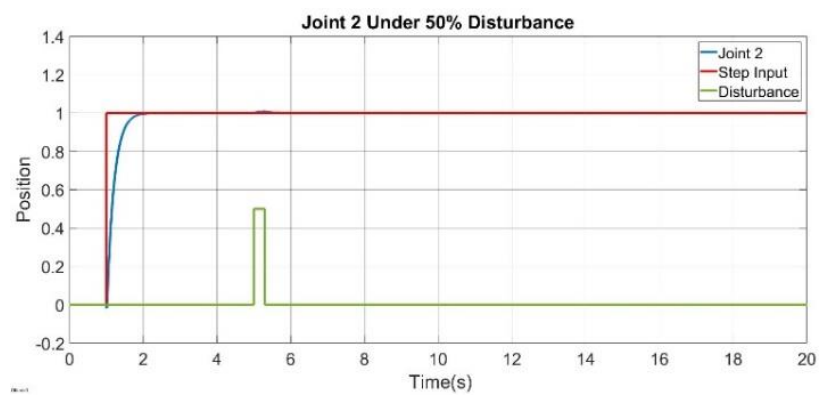

Fig. 11. SMC Under 50\% Disturbance

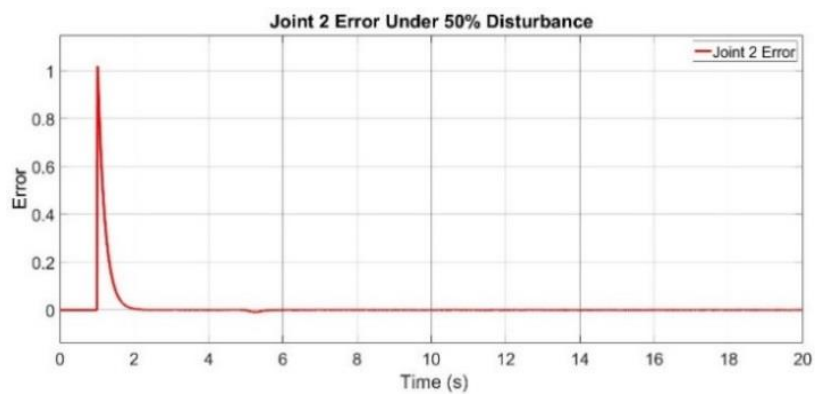

Fig. 12. Error for Joint 2

\section{SMC under $100 \%$ Disturbance}

To validate the response of SMC again, now disturbance of $100 \%$ is applied to joint 1 and 2 . SMC has found to be remain stable even with the $100 \%$ disturbance and settles the system within short period of time as shown below in Fig. 13 and Fig. 14. Maximum error of 0.0027 occurred at 6.7 seconds in joint 1 when disturbance acts on it as shown in Fig. 15. From Fig. 16 maximum error of 0.0027 is found at 6.6 seconds for joint 2 under the applied disturbance.

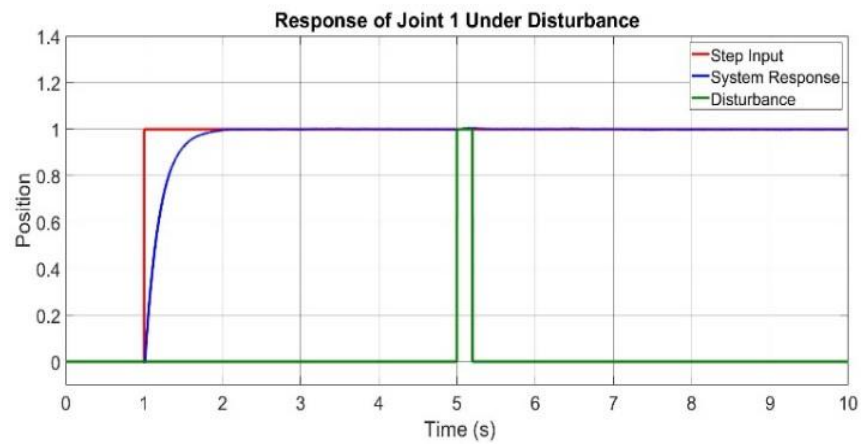

Fig. 13. Joint 1 Under Disturbance

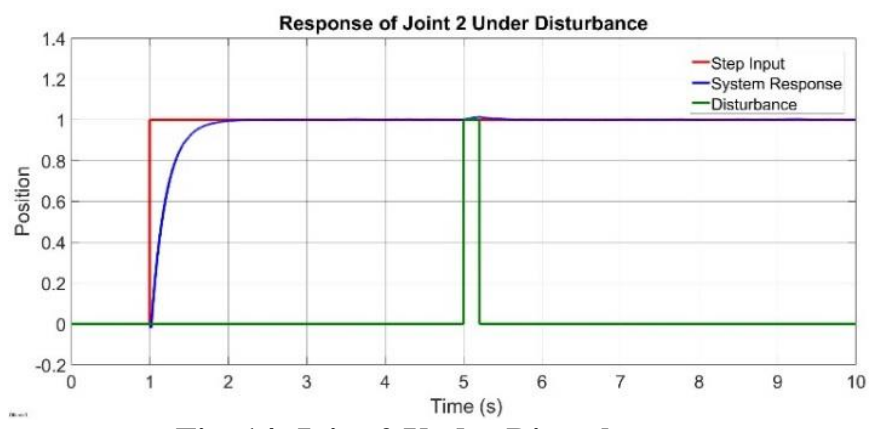

Fig. 14. Joint 2 Under Disturbance 
Sliding Mode Control for 2 Degrees of Freedom Upper Limb Rehabilitation Robotic System under Uncertainties

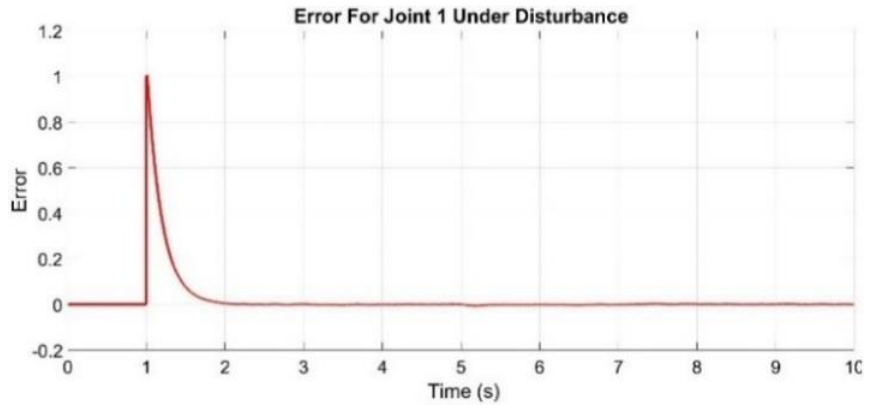

Fig. 15. Joint 1 Error Under Disturbance

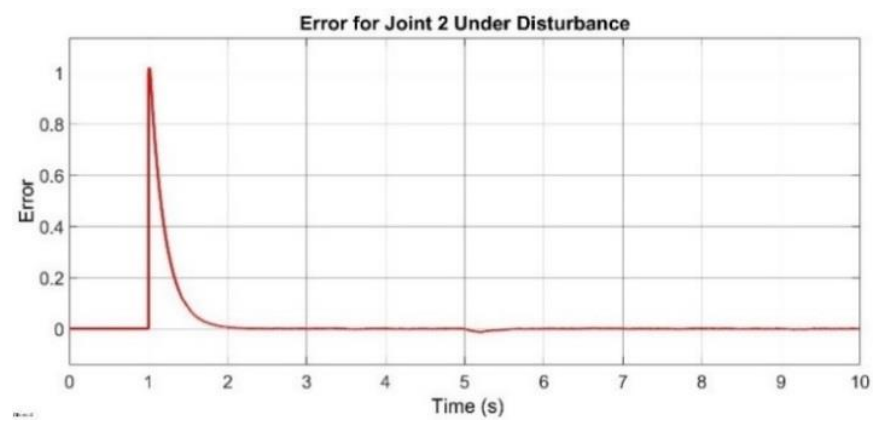

Fig. 16. Joint 2 Error Under Disturbance

\section{Comparison of SMC And MPC}

To highlight the performance of SMC, comparison between SMC and MPC is made by implementing them on the same mechanical structure of upper limb rehabilitation robotic system. Design methodology of MPC with all the results are provided in [36]. Below in Fig. 17 is the graph showing percent overshoot of the system response for MPC and Fig. 18 shows comparison between SMC and MPC under the action of $100 \%$ disturbance. This graph utterly shows the better performance of SMC as it has very little amount of percent overshoot than MPC. Overshoot in real therapy practice will cause the robot to move out of its pre-determined trajectory and cause damage and harm to patient. Hence the less the overshoot, the safer and more reliable the control is.

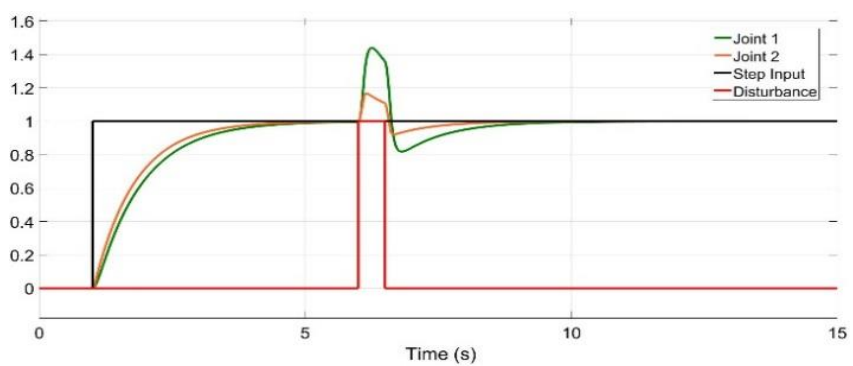

Fig. 17. MPC Under 100\% Disturbance

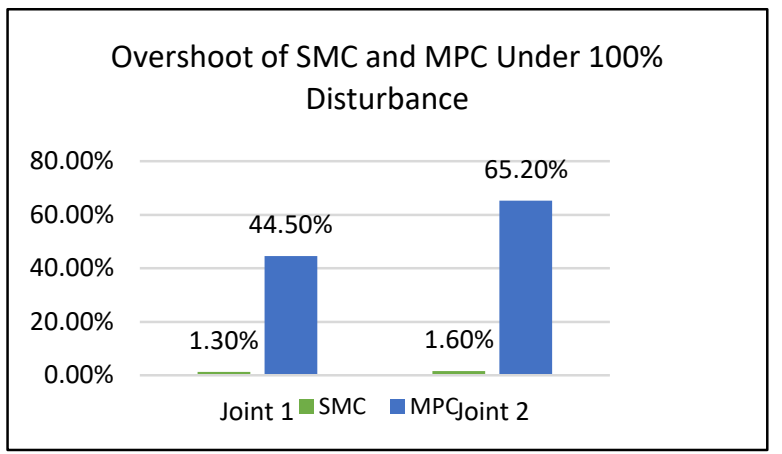

Fig. 18. Percent overshoot of SMC and MPC Under Disturbances

\section{ANALYSIS AND DISCUSSION}

From the above results and graphs, it has been found that sliding mode control is seems to be a robust and a stable controller for 2 DOF upper limb robotic manipulator. Conventional SMC produces chattering in the response of the system, which in practical implementation will cause oscillations in the arm and may cause harm to the patients undergoing rehabilitation. Reducing or eliminating this chattering is a vital part for the controller design. In this work, chattering has been reduced by applying boundary layer into the control input to make the response of the system smooth yet to ensure the safety and reliability of the robot to be used for therapy sake. In therapy movements, there may exist any sort of disturbance such as human-arm coupling, or presence of load torques in real world application so to validate the SMC under disturbed condition, 50\% and 100\% disturbance have been applied at 5 second for both joint 1 and 2 . The results have showed that SMC is capable of dealing with the disturbance in short period of time. Overshoot of the system response is found to be very low, $0.7 \%$ and $0.9 \%$ under $50 \%$ disturbance and $1.3 \%$ and $1.6 \%$ under $100 \%$ disturbance for joint 1 and joint 2 respectively. A comparison of MPC and SMC is made for the same mechanical structure under similar perturbed condition in which SMC proves to be more stable and efficient controller for the system.

\section{CONCLUSION}

This paper presents sliding mode control for 2 DOF upper limb rehabilitation robot. Kinematic and dynamic model of the arm robot is presented in this study. SMC is stable and robust control for controlling robots due to its handling capacities of model uncertainties and parametric changes. One drawback of using SMC is chattering, which can be reduced or eliminated by using boundary layer technique. This study shows the response of SMC with boundary layer and under the action of disturbances. Form the results it has been found that settling time of SMC is very short with very low percent overshoot, less than $2 \%$ even with $100 \%$ of disturbance and minimum steady state error. For justification and validation of the SMC, its results have been compared with that of MPC under same disturbed condition and a huge difference of overshoot between MPC and SMC has been found and clearly SMC proves to be more stable and efficient. Its means that sliding mode control is able to handle external disturbances significantly and thus proved to be a robust and stable controller which can be practically implemented on robotic manipulator to provide safe and reliable therapy movements.

\section{REFERENCES}

1. Owolabi, Mayowa O., The burden of stroke in Africa: a glance at the present and a glimpse into the future, Cardiovascular journal of Africa 26.2 H3Africa Suppl, 2015.

2. Johnson, Walter, Stroke: a global response is needed." Bulletin of the World Health Organization, Vol. 94, no. 9, pp.634, 2016.

3. Maciejasz, Paweł, A survey on robotic devices for upper limb rehabilitation, Journal of neuro engineering and rehabilitation, Vol. 11, no. 1, pp.3, 2014.

4. Platz, T, Evidenzbasierte Armrehabilitation Eine systematische Literaturubersicht, Der Nervenarzt, Vol. 74, no. 10, pp.841-849, 2003.

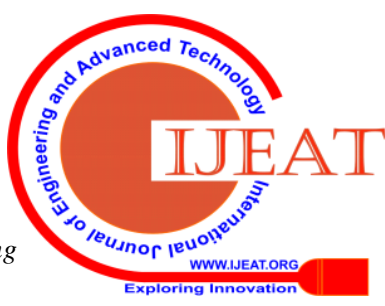


5. Feys, Hilde, Early and repetitive stimulation of the arm can substantially improve the long-term outcome after stroke: a 5-year follow-up study of a randomized trial, Stroke, Vol. 35, no. 4, pp.924-929, 2004.

6. Patton, James, Steven L. Small, and William Zev Rymer, Functional restoration for the stroke survivor: informing the efforts of engineers, Topics in stroke rehabilitation, Vol. 15, no. 6, pp.521-541, 2008.

7. Lo, Ho Shing, and Sheng Quan Xie, Exoskeleton robots for upper-limb rehabilitation: State of the art and future prospects, Medical engineering \& physics Vol. 34, no. 3, pp.261-268, 2012.

8. Huang, Vincent S., and John W. Krakauer, Robotic neurorehabilitation: a computational motor learning perspective, Journal of neuro engineering and rehabilitation Vol. 6, no. 1, pp.5 2009.

9. Laver, Kate, Virtual reality stroke rehabilitation-hype or hope?, Australian Occupational Therapy Journal, Vol. 58, no. 3, pp.215-219, 2011.

10.Nef, Tobias, Matjaz Mihelj, and Robert Riener, ARMin: a robot for patient-cooperative arm therapy, Medical \& biological engineering \& computing Vol 45, no. 9, pp.887-900, 2007.

11.Yu, Wen, and Jacob Rosen, A novel linear PID controller for an upper limb exoskeleton, Decision and Control (CDC), 2010 49th IEEE Conference on. IEEE, 2010.

12.Rahman, Mohammad H, Control of an exoskeleton robot arm with sliding mode exponential reaching law, International Journal of Control, Automation and Systems Vol. 11, no. 1, pp.92-104, 2013.

13.Rahman, Mohammad Habibur, Nonlinear control of an upper-limb exoskeleton robot, Electronics, Circuits and Systems (ICECS), 2011 18th IEEE International Conference on. IEEE, 2011.

14.Komada, Satoshi, Development of a biofeedback therapeutic-exercise-supporting manipulator, IEEE Transactions on Industrial Electronics Vol. 56, no. 10, pp.3914-3920 ,2009.

15.Bergamasco, Massimo, An arm exoskeleton system for teleoperation and virtual environments applications, Robotics and Automation, 1994. Proceedings., 1994 IEEE International Conference on. IEEE, 1994.

16.Kong, Kyoungchul, and Masayoshi Tomizuka, Control of exoskeletons inspired by fictitious gain in human model, IEEE/ASME Transactions on Mechatronics Vol. 14, no. 6, pp.689-698, 2009.

17.Yang, Yin, Model-based fuzzy adaptation for control of a lower extremity rehabilitation exoskeleton, Advanced Intelligent Mechatronics, 2009. AIM 2009. IEEE/ASME International Conference on. IEEE, 2009.

18.Gomes, Marciel A, Guilherme LM Silveira, and Adriano AG Siqueira, Gait-pattern adaptation algorithms based on neural network for lowe limbs active orthoses, Intelligent Robots and Systems, IROS 2009. IEEE/RSJ International Conference on. IEEE, 2009.

19.Slotine, Jean-Jacques E., and Weiping Li, Applied nonlinear control, Englewood Cliffs, NJ: Prentice hall, Vol. 199, no. 1, 1991.

20.Hung, Nguyen, Design of a sliding mode controller for an automatic guided vehicle and its implementation, International Journal of Control, Automation and Systems Vol. 8, no. 1, pp.81-90, 2010.

21.Tai, Nguyen Trong, and Kyoung Kwan Ahn, A RBF neural network sliding mode controller for SMA actuator, International Journal of Control, Automation and Systems Vol. 8, no. 6, pp.1296-1305, 2010.

22.Xu, Guohua, et al. "Trajectory tracking for underwater manipulator using sliding mode control." Robotics and Biomimetics, 2007. ROBIO 2007. IEEE International Conference on. IEEE, 2007.

23.Zhao, Dongya, Shaoyuan Li, and Quanmin Zhu, A new TSMC prototype robust nonlinear task space control of a 6 DOF parallel robotic manipulator, International Journal of Control, Automation and Systems Vol. 8, no. 6, pp.1189-1197, 2010.

24.Beyl, Pieter, A proof-of-concept exoskeleton for robot-assisted rehabilitation of gait, 4th European Conference of the International Federation for Medical and Biological Engineering. Springer, Berlin, Heidelberg, 2009.

25.Chang, Ming-Kun, An adaptive self-organizing fuzzy sliding mode controller for a 2-DOF rehabilitation robot actuated by pneumatic muscle actuators, Control Engineering Practice Vol. 18, no. 1, pp.13-22, 2010.

26.Slotine, Jean-Jacques E., and Weiping Li., Applied nonlinear control, Englewood Cliffs, NJ: Prentice hall, Vol. 199, no. 1, 1991.

27.H. K. Khalil, Nonlinear Systems, 3rd ed., Prentice Hall, Upper Saddle River, N.J., 2002.

28.Aschemann, Harald, and Dominik Schindele, Sliding-mode control of a high-speed linear axis driven by pneumatic muscle actuators, IEEE Transactions on Industrial Electronics Vol. 55, no. 11, pp.3855-3864, 2008.

29. J. J. Craig, Introduction to Robotics: Mechanics and Control, Upper Saddle River, NJ, USA: Prentice-Hall vol. 3, 2005.
30.Hemati, Neyram, and Ming-Chuan Leu, A complete mode characterization of brushless DC motors, IEEE Transactions on Industry Applications Vol. 28, no. 1, pp.172-180, 1992.

31.Brahmi, Brahim, Adaptive Tracking Control of an Exoskeleton Robot With Uncertain Dynamics Based on Estimated Time-Delay Control, IEEE/ASME Transactions on Mechatronics Vol. 23, no. 2, pp.575-585, 2018.

32.Rahman, Mohammad Habibur, Nonlinear control of an upper-limb exoskelton robot, Electronics, Circuits and Systems (ICECS), 2011 18th IEEE International Conference on. IEEE, 2011.

33.Djerioui, Ammar, Dehimi Ouali, and Mohamed Ladjal, Sliding Mode Control Using SVM for Power Quality Enhancement in Stand-Alone System Based on Four-Leg Voltage, International Journal of Intelligent Engineering and Systems 2018.

34.Khettab, Khatir, Yassine Bensafia, and Samir Ladaci, Chattering Elimination in Fuzzy Sliding Mode Control of Fractional Chaotic Systems Using a Fractional Adaptive Proportional Integral Controller, International Journal of Intelligent Engineering and Systems Vol. 10, no. 5, pp.255-265, 2017.

35.Bendjaima, Bachir, Djamel Saigaa, and Djalal Eddine Khodja, Fault Tolerant Control Based on Adaptive Fuzzy Sliding Mode Controller for Induction-Motors, International Journal of Intelligent Engineering and Systems Vol. 10, no. 3, pp.39-48, 2017.

36.Ali, Athar, et al, MPC-PID comparison for controlling therapeutic upper limb rehabilitation robot under perturbed conditions, Engineering Technologies and Social Sciences (ICETSS), 2017 IEEE 3rd International Conference on. IEEE, 2017.

\section{AUTHORS PROFILE}

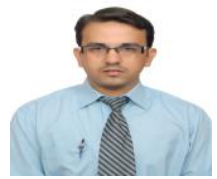

Syed Faiz Ahmed is the author of many research papers in the file of controls and robotics. His area of expertise includes, robotic, control \& embedded system

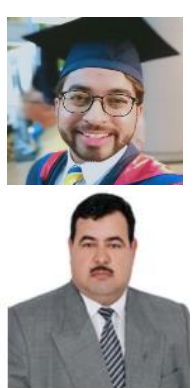

Yarooq Raza, completed his Masters in electrical \& electronic Engineering from University of Kuala Lumpur, Malaysia. His area of interest includes, robotics, optimal controls system designing

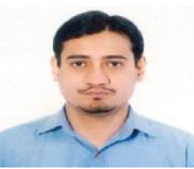

Muhammad Kamran Joyo completed his PHd in electrical Engineering from University of Kuala Lumpur, Malaysia. His area of interest includes, robotics, optimal controls system designing

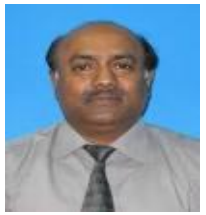

Asadullah Shah is the professor in international Islamic university Malaysia. Dr. Shah has a total of 30 years teaching and research experience. He has 180 research publications in International \& national journals and conference proceedings. 